\title{
Hypersensitivity to cisplatin after hRev3 mRNA knockdown in head and neck squamous cell carcinoma cells
}

\author{
MAKOTO ADACHI $^{1}$, KEI IJICHI $^{1,3}$, YASUHISA HASEGAWA ${ }^{2}$, TETSUYA OGAWA ${ }^{2}$, HIDEAKI NAKAMURA ${ }^{1}$, \\ YOSHIHIRO YASUI ${ }^{1}$, MASAKAZU FUKUSHIMA ${ }^{4}$ and KANJI ISHIZAKI ${ }^{1}$ \\ ${ }^{1}$ Central Laboratory and Radiation Biology, Aichi Cancer Center Research Institute, ${ }^{2}$ Department of Head and \\ Neck Surgery, Aichi Cancer Center Hospital, 1-1 Kanokoden, Chikusa-ku, Nagoya 464-8681; ${ }^{3}$ Department of \\ Otolaryngology - Head and Neck Surgery, Nagoya City University Graduate School of Medical Sciences, \\ 1 Kawasumi, Mizuho-cho, Mizuho-ku, Nagoya 467-8601; ${ }^{4}$ Tokushima Research Center, \\ Taiho Pharmaceutical Co., Ltd., 224-2 Tokushima-city, Tokushima 771-0194, Japan
}

Received March 28, 2008; Accepted June 5, 2008

DOI: $10.3892 / \mathrm{mmr} 00000015$

\begin{abstract}
Resistance to platinum-based chemotherapy frequently poses a significant problem in the treatment of head and neck squamous cell carcinomas (HNSCCs). In this study, we isolated cisplatin-resistant UM-SCC-23 CDDP/R cells from a UM-SCC-23 head and neck squamous cell carcinoma cell line. The UM-SCC-23 CDDP/R cells were approximately 3.5 -fold more resistant to cisplatin than the UM-SCC-23 cells. Translesion DNA synthesis (TLS) is one of the major pathways involved in post-replication repair. To ascertain whether TLS is involved in cisplatin resistance in human cancer cells, we analyzed expression of the Rev3 gene, which encodes the catalytic subunit of DNA polymerase $\xi$ (Polל), using quantitative PCR. Expression of hRev3 mRNA in the UM-SCC-23 CDDP/R cells was approximately 1.4-fold higher than in the UM-SCC-23 cells. In both types of cells, expression of hRev3 mRNA was suppressed using siRNAs. Cisplatin sensitivity after hRev3 mRNA knockdown increased approximately 2-fold in UM-SCC-23 cells and approximately 3 -fold in UM-SCC-23 CDDP/R cells. This study suggests that hRev3 knockdown with siRNAs increases sensitivity to cisplatin. Inhibition of the hRev3 gene may therefore prove to be a novel clinical strategy for reducing resistance to platinumbased chemotherapy in HNSCC.
\end{abstract}

Correspondence to: Dr Kei Ijichi, Department of Otolaryngology Head and Neck Surgery, Nagoya City University Graduate School of Medical Sciences, 1 Kawasumi, Mizuho-cho, Mizuho-ku, Nagoya 467-8601, Japan

E-mail: ijichi@med.nagoya-cu.ac.jp

Abbreviations: HNSCC, head and neck squamous cell carcinoma; Pol $\zeta$, DNA polymerase $\zeta$; TLS, translesion DNA synthesis; HR, homologous recombination

Key words: head and neck squamous cell carcinoma, cisplatin sensitivity, Rev3 gene, siRNA, DNA polymerase $\zeta$

\section{Introduction}

Cisplatin-based chemotherapy is widely used in the management of locally-advanced head and neck squamous cell carcinoma (HNSCC). However, recurrence often occurs following primary treatment, and treatment of recurrent tumors frequently fails due to resistance to therapeutic agents, including cisplatin (1). Although, to date, there have been many studies concerning the mechanisms of chemoresistance, effective strategies against chemoresistance in HNSCC (2-4) have yet to be devised.

The major cytotoxic effect of cisplatin is through the formation of DNA damage, such as DNA-interstrand crosslinks (4). After exposure to cisplatin, cells show one of two reactions: the induction of apoptosis through signal transduction, trigged by DNA damage and resulting in cell death, or the repair of DNA damage for cell survival (5).

Translesion DNA synthesis (TLS) and homologous recombination (HR) are the two major pathways involved in post replication repair (6). A number of TLS polymerases have been identified in Saccharomyces cerevisiae and mammals (7).

Candidates for translesion synthesis past bulky DNA adducts in vivo include members of the DNA polymerase $\mathrm{X}$ (polymerase $\mathrm{B}$, polymerase $\mu$, polymerase $\lambda$ ), polymerase B (polymerase $\xi$ ) and polymerase Y (Rev1, polymerase $\eta$, polymerase $\tau$, polymerase $\kappa$ ) families. Of these, DNA polymerase $\eta$ exhibits the highest translesion activity for the cisplatin adduct, followed by polymerase $\mu$ and polymerase $\zeta$ (8).

The Rev3 and Rev7 genes encode the catalytic subunit of DNA polymerase $\zeta$ (Pol $\zeta$ ) (7). Inactivation of the Rev3 gene sensitized chicken cells to cisplatin and to various DNA damaging agents, such as UV, methyl methanesulfonate and ionizing radiation. hRev3 antisense-expressing human fibroblasts demonstrated hypersensitivity to the cytotoxic effect of cisplatin (9). These results suggest that the subunit genes of Polל significantly affect cellular resistance to cisplatin. It may therefore be possible to overcome cisplatin resistance through the inhibition of these genes.

In this study, cisplatin-resistant UM-SCC-23 CDDP/R cells were established from cisplatin-sensitive UM-SCC-23 cells. 
Increased levels of hRev3 mRNA were found to be associated with cisplatin-resistant cells. Suppression of hRev3 mRNA in these cells resulted in increased sensitivity to cisplatin. Our data support the hypothesis that hRev3 is involved in the repair of cisplatin-induced DNA damage in human HNSCC cells.

\section{Materials and methods}

Cells and cell culture. The HNSCC UM-SCC-23 cell line was kindly donated by Dr Thomas E. Carey, Laboratory of Head and Neck Cancer Biology at the University of Michigan, USA. This cell line was maintained in Dulbecco's modified Eagle's medium (DMEM) (Sigma, St. Louis, MO, USA) supplemented with $10 \%$ fetal bovine serum (FBS) (Invitrogen, Carlsbad, CA, USA) in a humidified atmosphere of $5 \% \mathrm{CO}_{2}$ at $37^{\circ} \mathrm{C}$.

Isolation of cisplatin-resistant cells. UM-SCC-23 cells $\left(10 \times 10^{6}\right)$ were inoculated into a $10-\mathrm{cm}$ dish and cultured for $24 \mathrm{~h}$ in DMEM with 10\% FBS. Cells were then treated with cisplatin (Nihonkayaku, Tokyo, Japan) at a concentration of $0.5 \mu \mathrm{g} / \mathrm{ml}$ for $24 \mathrm{~h}$. Subsequently, cells were cultured in DMEM without cisplatin until the restoration of stable growth. The concentration of cisplatin treatment was stepwisely increased from 1.0 to 2.0, 3.0, 4.0 and $5.0 \mu \mathrm{g} / \mathrm{ml}$.

Analysis of hRev3 mRNA expression. Expression of hRev3 mRNA in the UM-SCC-23 and UM-SCC-23 CDDP/R cells was determined by real-time RT-PCR. Total RNA was extracted with Trizol reagent (Invitrogen) from the cell lines, and the initial cDNA strand synthesis was performed with ThermoScript ${ }^{\mathrm{TM}}$ (Invitrogen) for the detection of hRev3 mRNA. Forward (5'-GCTCCAGTATGTGTACCATCTTGT) and reverse (5'-ATGGATATCTCGAAGTAACACGTC) primers were used under the following conditions: $10 \mathrm{~min}$ at $95^{\circ} \mathrm{C}$ and 40 cycles of $5 \mathrm{sec}$ at $95^{\circ} \mathrm{C}, 20 \mathrm{sec}$ at $60^{\circ} \mathrm{C}$, and $40 \mathrm{sec}$ at $72^{\circ} \mathrm{C}$. The LightCycler System (Roche Diagnostics, Sandhoferstrasse, Mannheim, Germany) was used with SYBR-Green PCR Core reagents (PE Biosystems, Werrinton, UK). The expression levels of hRev3 mRNA for each sample were determined by standardization with expression levels of ß-actin.

Short interfering RNA transfection. siRNAs were synthesized to the target sequences of hRev3 mRNA corresponding to nt 860-879 (5'-GAUCACAGGUUUGUGCCAGtt) and 11901209 (5'-AGACUGAGUGAGUCACCUGtt) (Invitrogen). Each siRNA contained two additional 2'-deoxythymidine nt at the $3^{\prime}$ end. The selected sequence was screened by means of a BLAST search against all known human genes to verify that only human Rev3 mRNA was targeted. In addition, a randomly rearranged sequence of REV3 860, also obtained from Invitrogen, was used as control siRNA. siRNAs were complexed with the cationic lipid siFector (B-Bridge, CA, USA) in Opti-MEM medium (Invitrogen) as recommended by the suppliers. The siRNA-siFector complex was added to $6-\mathrm{cm}$ dishes at a final concentration of $2 \mu \mathrm{M}$. After $8 \mathrm{~h}$, cells were washed twice with PBS. Following transfection, hRev3 mRNA expression levels were evaluated by real-time RT-PCR at 24,48 and $72 \mathrm{~h}$.

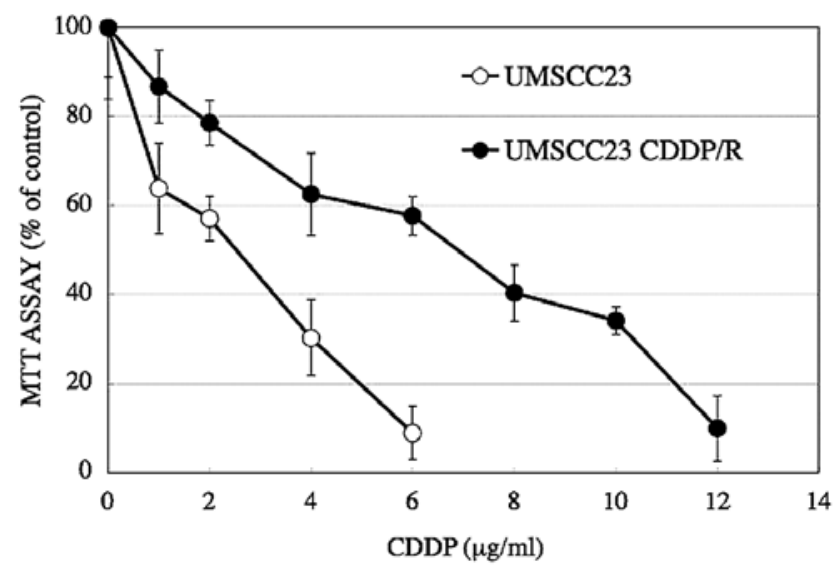

Figure 1. Cisplatin sensitivity of UM-SCC 23 cells and resistant derivative UM-SCC 23 CDDP/R cells. Both cell lines were treated with increasing concentrations of cisplatin for $24 \mathrm{~h}$, and cytotoxicity analysis was performed using the MTT assay. Each data point is the mean of three independent experiments. Vertical bars indicate standard deviations.

Tetrazolium dye (MTT) cytotoxicity assay. After siRNA treatment, cellular sensitivity to cisplatin and bleomycin was determined by the MTT cytotoxicity assay. Cells were plated in 48 -well plates at $2.0 \times 10^{4}$ cells/well and allowed to adhere for $24 \mathrm{~h}$, then siRNA-siFector complex was added to each well. Medium was replaced with 10\% FBS-DMEM $24 \mathrm{~h}$ after the addition of the siRNAs. The medium was removed $48 \mathrm{~h}$ later and replaced with fresh medium with or without increasing concentrations of cisplatin or bleomycin. Cells were then incubated for an additional $48 \mathrm{~h}$. Subsequently, the medium was replaced with fresh medium without cisplatin or bleomycin, and cells were incubated for a further $24 \mathrm{~h}$. Cell survival was then determined using Cell Proliferation Kit I (MTT) (Roche Applied Science, Mannheim, Germany). The experimental data represent the average of six replicates, and experiments were repeated at least three times.

\section{Results}

Cisplatin sensitivity. We analyzed the cisplatin sensitivity of the UM-SCC-23 cells and of the UM-SCC-23 CDDP/R cells isolated from the UM-SCC-23 cells using the MTT assay. The results are shown in Fig. 1. UM-SCC-23 CDDP/R cells were $\sim 3.5$-fold more resistant to cisplatin than UM-SCC-23 cells.

hRev3 mRNA expression levels. Expression of hRev3 mRNA in UM-SCC-23 and UM-SCC-23 CDDP/R cells was analyzed by real-time RT-PCR (Fig. 2). Expression levels of hRev3 mRNA in the UM-SCC-23 CDDP/R cells were $\sim 1.4$-fold higher than expression levels in the UM-SCC-23 cells.

Knockdown hRev3 expression by siRNAs. A decrease in hRev3 mRNA expression in both cell lines was induced by the introduction of the siRNAs (Fig. 3). Both types of cells treated with REV3 860 and REV3 1190 exhibited a 96-97\% suppression of hRev3 mRNA expression after $72 \mathrm{~h}$, while siRNA-free and control siRNA treatment caused no change in Rev3 expression. 


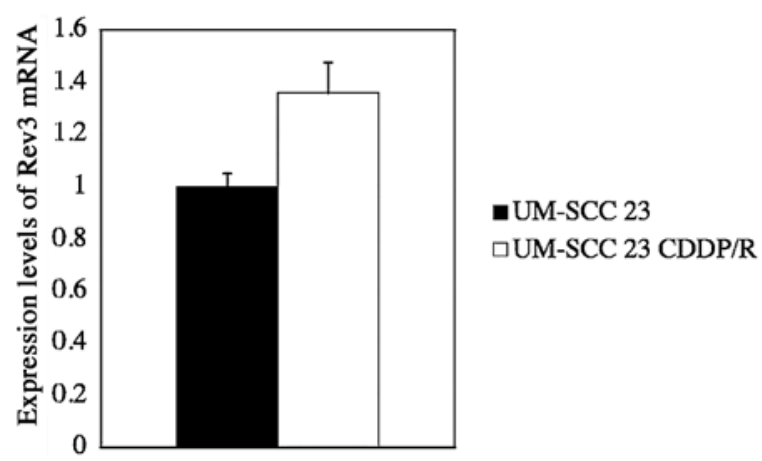

Figure 2. Relative expression of hRev3 mRNA in UM-SCC 23 CDDP/R cells compared to UM-SCC 23 cells. Expression of hRev3 mRNA was determined by real-time RT-PCR analysis. Each data point is the mean of three independent experiments.

A

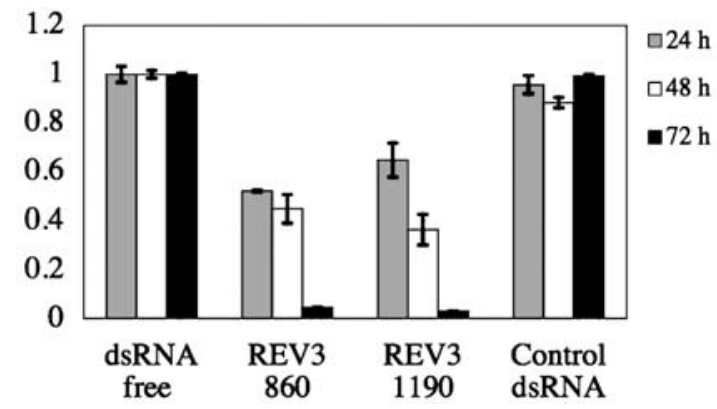

B

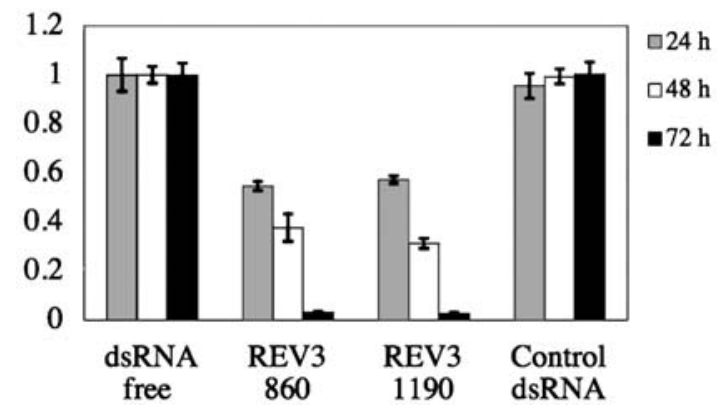

Figure 3. hRev3 mRNA expression after siRNA transfection. Relative expression of hRev 3 mRNA was determined at 24,48 and $72 \mathrm{~h}$ after siRNA transfection in UM-SCC 23 (A) and UM-SCC 23 CDDP/R (B) cells. siRNAs were synthesized to target sequences of hRev 3 mRNA corresponding to nt 860-879, REV3 860, and 1190-1209, REV3 1190. A randomly rearranged sequence of REV3 860 was used as control siRNAs. Each data point is the mean of three independent experiments.

Cisplatin sensitivity after hRev3 knockdown. Cisplatin sensitivity after suppressing hRev3 mRNA expression levels was determined using the MTT assay (Fig. 4). The hRev3 knockdown by REV3 860 and REV3 1190 caused an 2-fold higher cisplatin sensitivity in UM-SCC-23 cells compared to siRNAfree and control siRNA treated cells. Cisplatin sensitivity increased $\sim 3$-fold in the UM-SCC-23 CDDP/R cells. However, when bleomycin sensitivity was analyzed after suppression of hRev3 mRNA expression in the UM-SCC-23 and UM-SCC-23 $\mathrm{CDDP} / \mathrm{R}$ cells, no difference was observed in cell survival (Fig. 5).
A

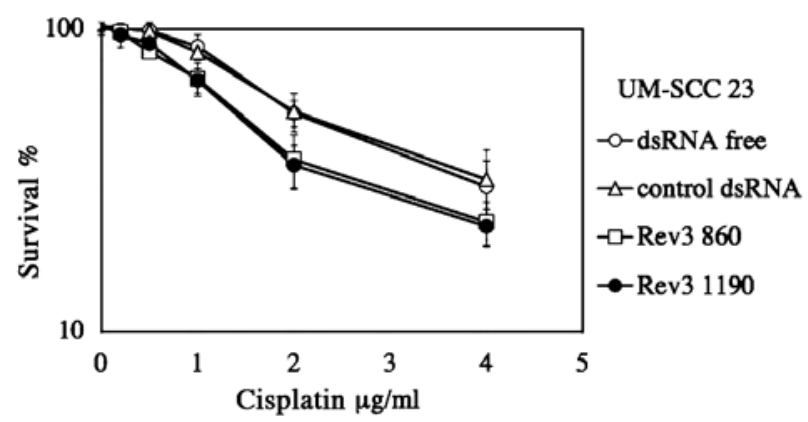

B

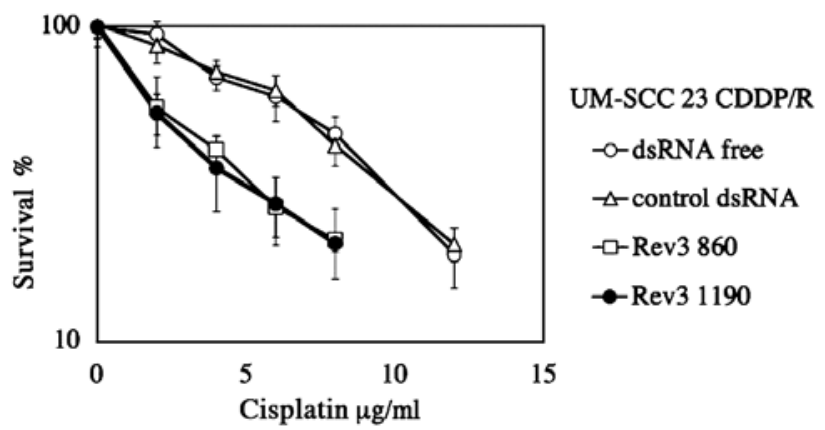

Figure 4. Cellular sensitivity to cisplatin after siRNA transfection. Sensitivity of UM-SCC 23 (A) and UM-SCC 23 CDDP/R (B) cells to cisplatin was determined by MTT cytotoxicity assay after cells were incubated for $24 \mathrm{~h}$ with or without siRNA. Each data point is the mean of three independent experiments.

A

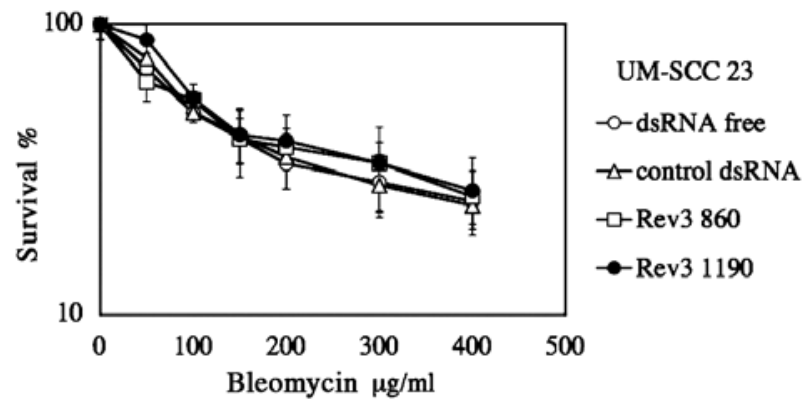

B

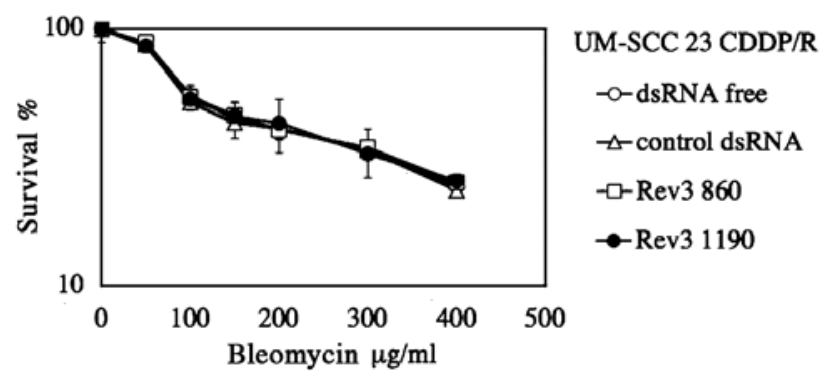

Figure 5. Cellular sensitivity to bleomycin after siRNA transfection. Sensitivity of UM-SCC 23 (A) and UM-SCC 23 CDDP/R (B) cells to bleomycin was determined by MTT cytotoxicity assay after cells were incubated for $24 \mathrm{~h}$ with or without siRNA. Each data point is the mean of three independent experiments. 


\section{Discussion}

In this study, we isolated the cisplatin-resistant cell line UMSCC-23 CDDP/R from cisplatin-sensitive UM-SCC-23 cells by treating cells with increasing concentrations of cisplatin.

The mechanisms of resistance to cisplatin have been investigated in previous studies. Based on the results of these studies, it has been suggested that Pol $\zeta$ activity is closely related to sensitivity to cisplatin. Homologous recombination and translesion DNA synthesis may contribute most to the repair of cisplatin damage, while Pol may be one of the most important TLS polymerases responsible for cisplatin tolerance.

Since Rev3 and Rev7 are the catalytic subunits of Pol $\zeta$, we determined hRev3 mRNA expression in these sensitive and resistant cell lines. As expected, expression in the resistant cells was higher than in the sensitive cells. This increase in hRev3 mRNA expression levels suggests that there is a relation between cisplatin resistance and hRev3 mRNA expression levels, consistent with a previous report that hRev3 mRNA expression was increased 2-fold after cisplatin treatment (9).

We additionally tried to suppress expression of hRev3 mRNA using specific siRNAs. In the two cell lines, hRev3 expression was effectively suppressed by siRNA transfection. After the knockdown of hRev3 expression, both cell lines had 2- to 3-fold increased sensitivity when compared to the control cells. This is consistent with the finding that Rev3 knockout mouse fibroblast cells showed an increase in cisplatin sensitivity (10). Thus, Polל plays an important role in DNA repair, which in turn plays a role in resistance to cisplatin.

In contrast, the sensitivity of bleomycin evidenced no change after treatment with siRNAs. The cytotoxicity of bleomycin is ascribed to the production of DNA double-strand breaks. The repair of these strand breaks is accomplished through homologous recombination or non-homologous end joining (11). The results of the present study suggest that Pols may not significantly contribute to such a repair of DNA double-strand breaks.

The results of the present study demonstrate that the change in hRev3 expression by molecular genetic methods is related to sensitivity to cisplatin in human cancer cells. These findings indicate that inhibition of the hRev3 gene on Pols may serve to enhance the efficacy of future cancer chemotherapy.

\section{Acknowledgements}

This study was supported in part by a collaborative study of the Aichi Cancer Center, Japan.

\section{References}

1. Bourhis J: New approaches to enhance chemotherapy in SCCHN. Ann Oncol 16 (Suppl 6): vi20-vi24, 2005.

2. Aoki K, Ogawa T, Ito Y and Nakashima S: Cisplatin activates survival signals in UM-SCC-23 squamous cell carcinoma and these signal pathways are amplified in cisplatin-resistant squamous cell carcinoma. Oncol Rep 11: 375-379, 2004.

3. Cullen KJ, Newkirk KA, Schumaker LM, Aldosari N, Rone JD and Haddad BR: Glutathione S-transferase pi amplification is associated with cisplatin resistance in head and neck squamous cell carcinoma cell lines and primary tumors. Cancer Res 63: 8097-8102, 2003.

4. Siddik ZH: Cisplatin: mode of cytotoxic action and molecular basis of resistance. Oncogene 22: 7265-7279, 2003

5. Ho YP, Au-Yeung SC and To KK: Platinum-based anticancer agents: innovative design strategies and biological perspectives. Med Res Rev 23: 633-655, 2003

6. Lawrence CW and Maher VM: Eukaryotic mutagenesis and translesion replication dependent on DNA polymerase zeta and Rev1 protein. Biochem Soc Trans 29: 187-191, 2001.

7. Gibbs PE, McGregor WG, Maher VM, Nisson P and Lawrence CW: A human homolog of the Saccharomyces cerevisiae REV3 gene, which encodes the catalytic subunit of DNA polymerase zeta. Proc Natl Acad Sci USA 95: 6876-6880, 1998.

8. Vaisman A, Lim SE, Patrick SM, Copeland WC, Hinkle DC, Turchi JJ and Chaney SG: Effect of DNA polymerases and high mobility group protein 1 on the carrier ligand specificity for translesion synthesis past platinum-DNA adducts. Biochemistry 38: 11026-11039, 1999.

9. Wu F, Lin X, Okuda T and Howell SB: DNA polymerase zeta regulates cisplatin cytotoxicity, mutagenicity, and the rate of development of cisplatin resistance. Cancer Res 64: 8029-8035, 2004.

10. Zander L and Bemark M: Immortalized mouse cell lines that lack a functional Rev3 gene are hypersensitive to UV irradiation and cisplatin treatment. DNA Repair (Amst) 3: 743-752, 2004.

11. Esposito G, Godindagger I, Klein U, Yaspo ML, Cumano A and Rajewsky K: Disruption of the Rev31-encoded catalytic subunit of polymerase zeta in mice results in early embryonic lethality. Curr Biol 10: 1221-1224, 2000. 